\title{
ANALYSIS, DIAGNOSIS AND PROGNOSIS OF LEPROSY UTILIZING FUZZY CLASSIFIER
}

\author{
*Obi J.C. ${ }^{1}$ and Imianvan A.A. ${ }^{2}$ \\ Department of Computer Science, University of Benin, Benin City. Nigeria. \\ *Correspondence author: tripplejo2k2@yahoo.com, +234(0)8093088218
}

\begin{abstract}
Leprosy (Hensen's Disease) is an infectious disease that progressively deteriorates over time and is associated with mycobacterium leprae (M.leprae). Although human-to-human transmission is the primary source of infection, three other species can carry and (rarely) transfer M.leprae to humans: chimpanzees, mangabey monkeys, and nine-banded armadillos. The early signs and symptoms of leprosy are very subtle and occur slowly (usually over years), which includes; numbness (loss of temperature sensation), skin lesions, weakness, anesthesia, paralysis, epistaxis, skin bumps, deformity (face, hand and foot), eye damage (dryness and reduced blinking), loss of digit, enlarged earlobes, conjunctivitis and Iridocyclitis. In this paper, the traditional procedure of the medical diagnosis of Leprosy employed by physicians is expressed using Fuzzy classifier. The proposed expert system eliminates uncertainty and imprecision associated with the diagnosis of Leprosy.

Keywords: Leprosy, Fuzzy Set, Fuzzy Logic, Fuzzy Classifier, Diagnosis
\end{abstract}

\section{INTRODUCTION}

Leprosy (Hensen's Disease) is chronic, progressive infectious disease caused by mycobacterium leprae (M.leprae) which causes skin sores and also affects the eyes, mucous membranes and peripheral nerves, MedicineNet (2011) and RightDiagnosis (2011). Leprosy develops slowly (from six months to 40 years) and results in skin lesions and deformities, most often affecting the cooler places on the body (for example, eyes, nose, earlobes, hands, feet and testicles). Although human-to-human transmission is the primary source of infection, three other species can carry and (rarely) transfer $M$. leprae to humans: chimpanzees, mangabey monkeys, and nine-banded armadillos, MedicineNet (2011).

The early signs and symptoms of leprosy are very subtle and occur slowly (usually over years), which includes; numbness (loss of temperature sensation), skin lesions, weakness, anesthesia, paralysis, epistaxis, skin bumps, deformity (face, hand and foot), eye damage (dryness and reduced blinking), loss of digit, enlarged earlobes, conjunctivitis and Iridocyclitis, Healthline (2011); MedicineNet (2011) and RightDiagnosis (2011).

There are different types of leprosy described in different literatures, Medicinenet (2011). These types are based on the person's immune response to M.leprae, which include:

a. Indeterminate leprosy in few hypopigmented macules which can heal spontaneously persists or advances to other forms.

b. Tuberculoid leprosy is usually associated with few hypo-pigmented macules, some are large and some become anesthetic (lose pain sensation or a substance that causes lack of feeling or awareness), some neural involvement in which nerves become enlarged, spontaneous resolution in a few years, persists or advances to other forms. c. Borderline tuberculoid leprosy is closely linked with lesions like tuberculoid leprosy but smaller and more numerous with less nerve enlargement, this form may persist, revert to tuberculoid leprosy, or advance to other forms.

d. Mid-borderline leprosy forms many reddish plaques that are asymmetrically distributed, moderately anesthetic, with regional adenopathy (swollen lymph nodes); the form may persist, regress to another form, or progress

e. Borderline lepromatous leprosy usually has many skin lesions with macules (flat lesions), papules (raised bumps), plaques and nodules, sometimes with or without anesthesia; the form may persist, regress or progress to lepromatous leprosy.

f. Lepromatous leprosy has early lesions which are pale macules (flat areas) that are diffuse and symmetric; later many M.leprae organisms can be found in them. Alopecia (hair loss) occurs; often patients have no eyebrows or eyelashes. As the disease progresses, nerve involvement leads to anesthetic areas and limb weakness; progression leads to aseptic necrosis (tissue death from lack of blood to area), lepromas (skin nodules), and disfigurement of many areas including the face. The lepromatous form does not regress to the other less severe forms. Histoid leprosy is a clinical variant of lepromatous leprosy that presents with clusters of histiocytes (a type of cell involved in the inflammatory response) and a grenz zone (an area of collagen separating the lesion from normal tissue) seen in microscopic tissue sections. 
Advance researchers over the years have suggested that M.leprae are spread person to person by nasal secretions or droplets. They speculate that infected droplets reach other peoples' nasal passages and begin the infection there. Infected droplets can infect others by entering breaks in the skin. Mycobacterium leprae apparently cannot infect intact skin. Rarely, humans get leprosy from the few animal species mentioned above. Occurrence in animals makes it difficult to eradicate leprosy from these endemic sources. Routes of transmission are still being researched for leprosy. Recent genetic studies have demonstrated that several genes (about seven) are associated with an increased susceptibility to leprosy; some researchers now conclude that susceptibility to leprosy may be partially inheritable, Healthline (2011) and MedicineNet (2011).

Existing methods employed by physicians for the diagnosis of leprosy uses manual methods characterized by the inability to handle uncertain or vague data existing between intervals. This paper has chosen to solve this problem by employing the rich facilities of fuzzy classifier in analyzing and diagnosis of Leprosy.

The majority of cases of leprosy are diagnosed by clinical findings, especially since most current cases are diagnosed in areas that have limited or no laboratory equipment available, Healthline (2011) and RightDiagnosis (2011). Hypo-pigmented patches of skin or reddish skin patches with loss of sensation, thickened peripheral nerves, or both clinical findings together often comprise the clinical diagnosis. Skin smears or biopsy material that show acid-fast bacilli with the Ziehl-Neelsen stain or the acid-fast stain (biopsy) can diagnose Multi-bacillary leprosy, or if bacteria are absent, diagnose Pauci-bacillary leprosy. Lepromin test, Phenolic glycolipid-1 test, Polymerase Chain Reaction (PCR), and Lymphocyte Migration Inhibition Test or (LMIT). Other tests such as CBC (Complete blood count) test, liver function tests, Creatinine test (urine test), or a nerve biopsy may be done to help determine if other organ systems have been affected, MedicineNet (2011).

Expert systems are knowledge-based systems that contain expert knowledge. An expert system is a program that can provide expertise for solving problems in a defined application area in the way the experts do. They use human knowledge to solve problems that normally would require human intelligence. These expert systems represent the expertise knowledge as data or rules within the computer. These rules and data can be called upon when needed to solve problems, PCAI (2002).

Fuzzy systems are rule-based expert systems based on fuzzy rules and fuzzy inference. Fuzzy sets were introduced by, Zadeh (1965) to represent/manipulate data and information possessing non statistical uncertainties. Fuzzy sets provide a means of representing and manipulating data that are not precise, but rather fuzzy. Fuzzy logic is a superset of conventional (Boolean) logic that has been extended to handle the concept of partial truth - truth values between "completely true" and "completely false", Christos and Dimitros (2008); Kasabov (1998) and Robert (2000). The theory of fuzzy logic provides a mathematical strength to capture the uncertainties associated with human cognitive processes, such as thinking and reasoning. A fuzzy set $A$ is called trapezoidal fuzzy number (Figure 1) with tolerance interval $[a, b]$, left width $a$ and right width $\beta$ if its membership function has the following form

$$
A(t)= \begin{cases}1-(a-t) / \alpha & \text { if } a-\alpha \leq t \leq a \\ 1 & \text { if } a \leq t \leq b \\ 1-(t-b) / \beta & \text { if } a \leq t \leq b+\beta \\ 0 & \text { otherwise }\end{cases}
$$

and we use the notation $A=(a, b, a, \beta)$. It can easily be shown that

$$
\begin{gathered}
{[A]^{\gamma}=[a-(1-\gamma) a, b+(1-\gamma) \beta], \forall y \varepsilon[0,1] .} \\
\text { The support of } A \text { is }(a-a, b+\beta) .
\end{gathered}
$$

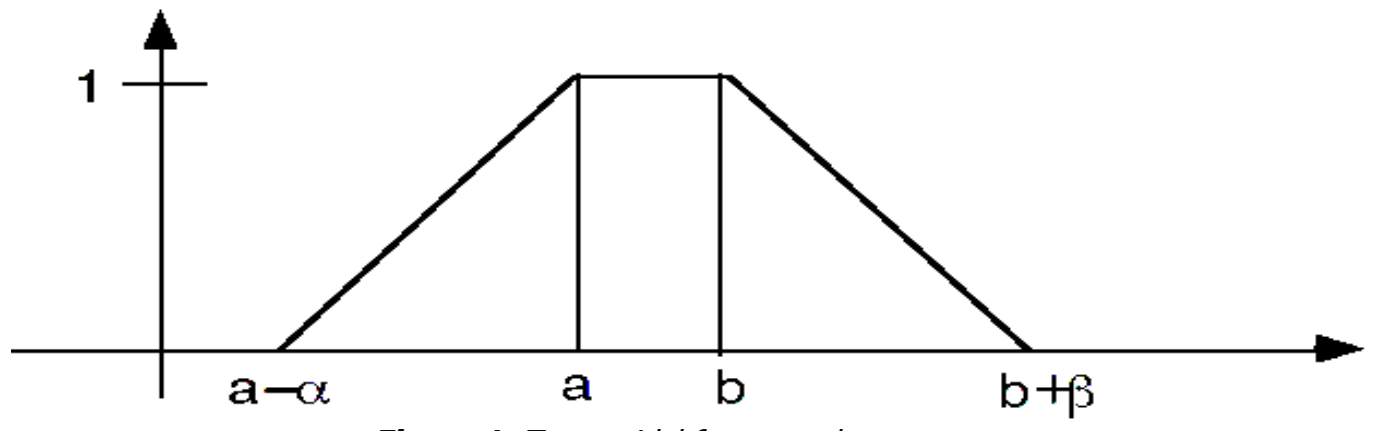

Figure 1: Trapezoidal fuzzy number

Fuzzy systems often learn their rules from experts. When no expert gives the rules, adaptive fuzzy systems learns by observing how people regulate real systems, Leondes (2010). The difference between classical and fuzzy logic is something called "the law of excluded middle", Bart and Satoru (1993). 
In standard set theory, if object does or does not belong to a set. There is no middle ground. In such bivalent systems, an object cannot belong to both its set and its compliment set or to neither of them. This principle preserves the structure of the logic and avoids the contradiction of object that both is and is not a thing at the same time, Zadeh (1965). However, fuzzy logic is highly abstract and employs heuristic (experiment) requiring human experts to discover rules about data relationship.

Fuzzy classification assumes the boundary between two neighboring classes as a continuous, overlapping area within which an object has partial membership in each class. It not only reflects the reality of many applications in which categories have fuzzy boundaries, but also provides a simple representation of the potentially complex partition of the feature space. Sun and Jang (1993) propose an adaptivenetwork-based fuzzy classifier to solve fuzzy classification problems. Conventional approaches of pattern classification involve clustering training samples and associating clusters to given categories. The complexity and limitations of previous mechanisms are largely due to the lacking of an effective way of defining the boundaries among clusters. This problem becomes more intractable when the number of features used for classification increases, Christos and Dimitros (2008); Kasabov (1998); Robert (2000) and Rudolf (2008).

\section{MATERIALS AND METHODS}

Fuzzy classifier is applied to the diagnosis of leprosy using the model prescribed in Figure 2. The system is developed in an environment characterized by Microsoft Windows XP Professional Operating System, Microsoft Access Database Management system, Visual Basic Application Language and Microsoft Excel. The Visual Basic provides the Interface for the application. The Interface Includes Login Form, Registration Form and authorized diagnosis form. The login session ensures that only authorized users' details which include name, sex, age, blood group, genotype, weight etc. The diagnosis page provides list of symptoms of gonorrhea which the user is expected to select the available options. The user expresses the intensity of each symptom by selecting options provided. The Inference Engine of the System utilizes the data provided by user for the diagnosis. The result of each complete diagnosis goes to the database (Microsoft Access database). The Microsoft Excel is used to present graphical expression of the result for decision making purposes. The Visual Basic, Microsoft Access Database Management System and Excel operate over the Microsoft Window XP backend engine which serves as the operating system for the application.

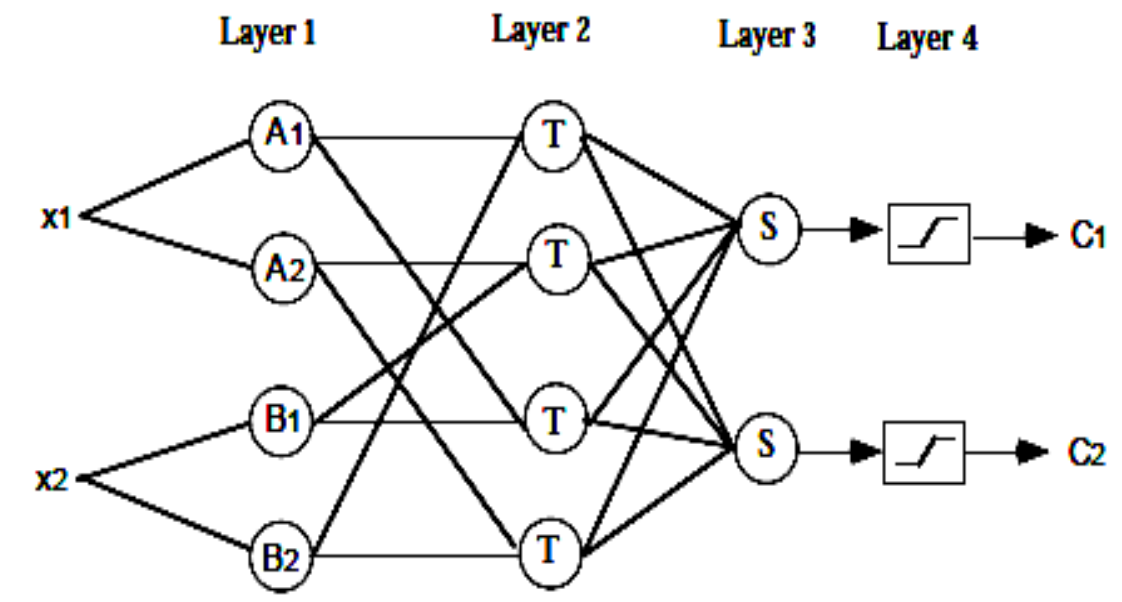

Figure 2: Fuzzy Classifier System for the Diagnosis of Leprosy

The system parades two input variables $X_{1}$ and $X_{2}$ which are symptoms of leprosy. The training data are categorized by two classes $C_{1}$ and $C_{2}$. Each input is represented by the two linguistic terms, thus we have four rules.

$$
\begin{aligned}
& A_{\mathrm{i}}(\mathrm{u})=\exp \left[-1 / 2\left(\mathrm{u}-\mathrm{a}_{\mathrm{i} 1} / \mathrm{b}_{\mathrm{i} 1}\right)^{2}\right], \\
B_{\mathrm{i}}(\mathrm{v})= & \exp \left[-1 / 2\left(\mathrm{v}-\mathrm{a}_{\mathrm{i} 2} / \mathrm{b}_{\mathrm{i} 2}\right)^{2}\right]
\end{aligned}
$$

which represent the linguistic terms, where $\left(a_{i 1}, a_{i 2}\right.$, $\left.b_{i 1}, b_{i 2}\right)$ is the parameter set. As the values of these parameters change, the bell-shaped functions vary accordingly, thus exhibiting various forms of membership functions on linguistic labels $A_{i}$ and $B_{i}$. In fact, any continuum, such as trapezoidal and triangular-shaped membership functions are also quantified candidates for node functions in this layer. The initial values of the parameters are set in such a way that the membership functions along each axis satisfy; completeness, normality and convexity. The parameters (symptoms) are then tuned with a descent-type method.

Layer 2: Each node generates the signal corresponding to the conjunctive combination of individual degrees of match of leprosy symptoms. The output signal is the firing strength of the fuzzy rule with respect to leprosy. 
We take the linear combination of the firing strengths of the rules at Layer 3 and apply sigmoidal function at Layer 4 to calculate the degree of belonging to a certain class. Fuzzy classification inference procedure is presented in Figure 3.

Given training set $\left\{\left(x^{k}, y^{k}\right), k=1 \ldots K\right\}$ where $x^{k}$ refers to the $k^{\text {th }}$ input pattern then

$$
Y^{K}=(1,0)^{\top} \text { If } X^{K} \text { belongs to Class } 1
$$

The error function for pattern $\mathrm{k}$ can be defined by

$$
E_{K}=1 / 2\left[\left(0_{1}{ }^{K}-Y_{1}{ }^{K}\right)^{2}+\left(0_{2}{ }^{K}-Y_{2}^{K}\right)^{2}\right]
$$

where $\mathrm{y}^{\mathrm{k}}$ is the desired output and $\mathrm{o}^{\mathrm{k}}$ is the computed output.

Using fuzzy IF-THEN rules to describe a classifier, assume that $K$ patterns $x_{p}=\left(x_{p 1}, x_{p n}\right)$,

$$
P=1, \ldots, K \text { are given from two classes, where } x_{p} \text { is an } n \text {-dimensional crisp vector. }
$$

IF $x_{p 1}$ is small and $x_{p 2}$ is very large THEN $x_{p}=\left(x_{p 1}, x_{p 2}\right)$ belongs to Class $C_{1}$

IF $x_{p 1}$ is large and $x_{p 2}$ is very small THEN $x_{p}=\left(x_{p 1}, x_{p 2}\right)$ belongs to Class $C_{2}$

Where $x_{p 1}$ and $x_{p 2}$ are the features of pattern (or object) $p$, small and very large are linguistic terms characterized by appropriate membership functions

Figure 3: Typical fuzzy classification rules for $n=2$

The task of fuzzy classification of leprosy is to generate an appropriate fuzzy partition of the feature space. In this context the word appropriate means that the number of misclassified patterns is very small or zero. Then the rule base should be optimized by deleting rules which are not used. The scheme is extensible to any number of input and classes.

\section{RESULTS AND DISCUSSION}

The fuzzy partition for each input feature consists of leprosy symptoms (numbness (loss of temperature sensation), skin lesions, weakness, anesthesia, paralysis, epistaxis, skin bumps, deformity (face, hand and foot), eye damage (dryness and reduced

\section{The Fuzzy IF-THEN Rules $\left(R_{i}\right)$ for Leprosy is}

R1: IF the patient is experiencing numbness (loss of temperature sensation) THEN patient is in class $\mathrm{C}_{3}$.

R2: IF the patient is experiencing numbness (loss of temperature sensation) and skin lesions THEN patient is in class $\mathrm{C}_{3}$.

R3: IF the patient is experiencing numbness (loss of temperature sensation), skin lesions and weakness THEN patient is in class $C_{3}$.

R4: IF the patient is experiencing numbness (loss of temperature sensation), skin lesions, weakness and anesthesia THEN patient is in class $\mathrm{C}_{1}$.

R5: IF the patient is experiencing numbness (loss of temperature sensation), skin lesions, weakness, anesthesia and paralysis THEN patient is in class $\mathrm{C}_{2}$.

R6: IF the patient is experiencing numbness (loss of temperature sensation), skin lesions, weakness, anesthesia, paralysis and epistaxis THEN patient is in class $\mathrm{C}_{2}$.

R7: IF the patient is experiencing numbness (loss of temperature sensation), skin lesions, weakness, anesthesia, paralysis, epistaxis and skin bumps THEN he/she has class $\mathrm{C}_{2}$.

R8: IF the patient is experiencing numbness (loss of temperature sensation), skin lesions, weakness, anesthesia, paralysis, epistaxis, skin bumps and deformity (face, hand and foot) THEN patient is in class $\mathrm{C}_{2}$.

R9: IF the patient is experiencing numbness (loss of temperature sensation), skin lesions, weakness, anesthesia, paralysis, epistaxis, skin bumps, deformity (face, hand and foot) and eye damage (dryness and reduced blinking) THEN patient is in class $\mathrm{C}_{2}$.

R10: IF the patient is experiencing numbness (loss of temperature sensation), skin lesions, weakness, anesthesia, paralysis, epistaxis, skin bumps, deformity (face, hand and foot), eye damage (dryness and reduced blinking) and loss of digit THEN patient is in class $\mathrm{C}_{2}$.

R11: IF the patient is experiencing numbness (loss of temperature sensation), skin lesions, weakness, anesthesia, paralysis, epistaxis, skin bumps, deformity (face, hand and foot), eye damage (dryness and reduced blinking), loss of digit, and enlarged earlobes THEN patient is in class $\mathrm{C}_{2}$.

R12: IF the patient is experiencing numbness (loss of temperature sensation), skin lesions, weakness, anesthesia, paralysis, epistaxis, skin bumps, deformity (face, hand and foot), eye damage (dryness and reduced blinking), loss of digit, enlarged earlobes and conjunctivitis THEN patient is in class $\mathrm{C}_{2}$.

R13: IF the patient is experiencing numbness (loss of temperature sensation), skin lesions, weakness, anesthesia, paralysis, epistaxis, skin bumps, deformity (face, hand and foot), eye damage (dryness and 
reduced blinking), loss of digit, enlarged earlobes, conjunctivitis and Iridocyclitis THEN patient is in class $\mathrm{C}_{2}$.

Leprosy prognosis is very severe if any patience is diagnosed with Cluster $2\left(\mathrm{C}_{2}\right)$, the patient should consult a physician immediately to prevent total deterioration.

The degree of intensity of leprosy symptoms ordered in the following classes for a typical scenario is presented in Table 1. From Table 1, Cluster 1 represent possible situation of "Might be leprosy" because at least four of the symptoms are pronounced. Cluster 2, represent possible situation of "Having Leprosy" since five or more of the symptoms is pronounced. Cluster 3 represent possible situation of "Not Leprosy" but possibly some other disease since only two of the symptoms of leprosy is pronounced.

Table 1: Degree of Membership of Leprosy Symptoms; Scale (0.00 -1.00)

\begin{tabular}{lcccc}
\hline $\begin{array}{l}\text { Symptoms (Parameters) } \\
\text { of Leprosy }\end{array}$ & $\begin{array}{c}\text { Leprosy } \\
\text { Symptom } \\
\text { Codes }\end{array}$ & $\begin{array}{c}\text { Degree of } \\
\text { Cluster } \\
\left(\mathbf{c}_{\mathbf{1}}\right)\end{array}$ & $\begin{array}{c}\text { Membership of Leprosy } \\
\text { Cluster } \\
\left(\mathbf{C}_{\mathbf{2}}\right)\end{array}$ & $\begin{array}{c}\text { Cluster } \\
\left(\mathbf{C}_{\mathbf{3}}\right)\end{array}$ \\
\hline Numbness (Loss of temperature & $\mathrm{P} 01$ & 0.20 & 0.30 & 0.50 \\
sensation) & & & & \\
Skin lesions & $\mathrm{P} 02$ & 0.10 & 0.30 & 0.60 \\
Weakness & $\mathrm{P} 03$ & 0.25 & 0.60 & 0.15 \\
Anesthesia & $\mathrm{P} 04$ & 0.25 & 0.50 & 0.25 \\
Paralysis & $\mathrm{P} 05$ & 0.22 & 0.70 & 0.08 \\
Epistaxis & $\mathrm{P} 06$ & 0.20 & 0.80 & 0.00 \\
Skin bump & $\mathrm{P} 07$ & 0.27 & 0.53 & 0.20 \\
Deformity (face, hand and foot) & $\mathrm{P} 08$ & 0.30 & 0.65 & 0.05 \\
Eye damage (dryness and reduced & $\mathrm{P} 09$ & 0.10 & 0.80 & 0.10 \\
blinking & & & & \\
Loss of digit, & $\mathrm{P} 10$ & 0.56 & 0.44 & 0.00 \\
Enlarged earlobes & $\mathrm{P} 11$ & 0.80 & 0.15 & 0.05 \\
Conjunctivitis & $\mathrm{P} 12$ & 0.50 & 0.37 & 0.13 \\
Iridocyclitis & $\mathrm{P} 13$ & 0.70 & 0.15 & 0.15 \\
Results & & Might be & Having & Not \\
& & Leprosy & Leprosy & Leprosy \\
\hline
\end{tabular}

Degree of Membership Function

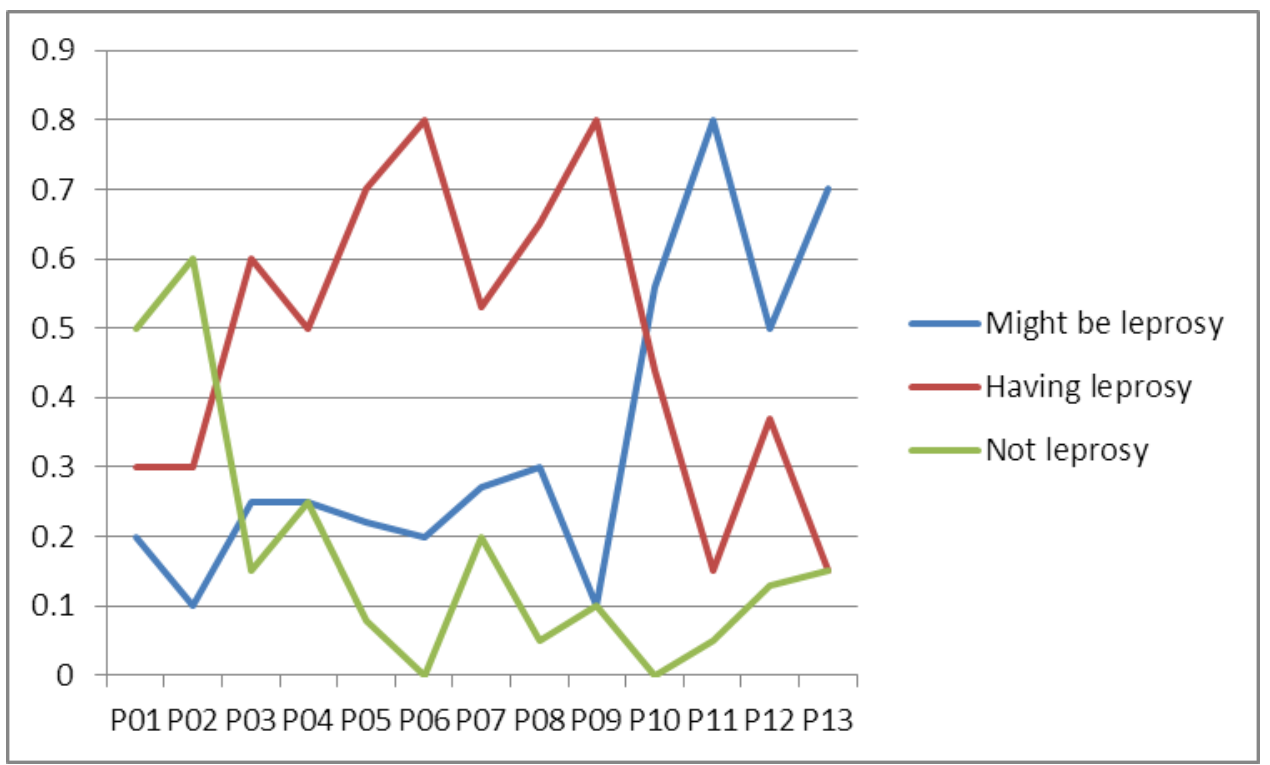

Figure 4: Graphical representation of Membership Grades of Leprosy symptoms

The graphical representation in Figure 4 , is a representation of Table 1 and clearly show four symptoms with high degree of "Might be Leprosy" in
Cluster 1, seven symptoms with high degree of "Having leprosy" in Cluster 2 and two symptoms with high degree of "Not having Leprosy" in Cluster 3. 


\section{CONCLUSION}

This work demonstrates the application of Information and Communication Technology (ICT) in the domain of differential diagnosis of Leprosy utilizing fuzzy classifier method when given a set of symptoms. Utilizing fuzzy classifier methodology, differential diagnosis of leprosy is categorized into three main groups "Might be Leprosy", "Having leprosy" and Not Leprosy" were presented. The system is designed to

\section{REFERENCES}

Bart K. and Satoru I. (1993), "Fuzzy Logic", retrieved from http//:Fortunecity .com/emachines/e11/86/fuzzylog.html.

Christos S. And Dimitros S. (2008) "Neural Network", retrieved from http://www.docstoc.com/docs/15050/neuralnetworks.

Healthline, (2011), "Leprosy, symptoms and causes", retrieved from http://healthline.com

Kasabov N. K. (1998), "Foundations of neural networks, fuzzy systems, and knowledge engineering", A Bradford Book, The MIT Press Cambridge, Massachusetts London, England

Leondes C. (2010), "The Technology of Fuzzy Logic Algorithm retrieved from Suite101.com/examples-of-expert-Systemapplication-in-artificial Intelligence.

MedicineNet, (2011), "Leprosy: Symptoms, causes and treatment", retrieved from http:// medicineNet.com diagnose leprosy and not to prescribe drugs or treatment but can be expanded to do so in future research. Soon there would be a fully computerized system to handle diagnosis of diseases in general. A system of this nature that has the ability to diagnose a person suffering from leprosy should be introduced in the health sector to assist doctors in making diagnosis most especially in cases of severe illnesses.

PCAI, (2002), "Expert Systems", retrieved from http:// pcai.com/web/al_info.

Robert F. (2000), "Introduction to Neuro-Fuzzy Systems, Advances in Soft Computing Series", Springer-Verlag, Berlin/Heildelberg, 289 pages

Rudolf K. (2008), Neuro-fuzzy systems, retrieved from http://ask.com/Neuro-fuzzy systems.

Sun C.T. and Jang J.S. (1993) "A neuro-fuzzy classifier and its applications", in: Proc. IEEE Int. Conference on Neural Networks, San Francisco, pp.94-98.

RightDiagnosis, (2011), "Introduction; Causes, diagnosis, types and treatment of Leprosy" retrieved from http:// wrongdiagnosis.com.

Zadeh L.A. (1965): "Fuzzy sets and systems". In: Fox J, editor, System Theory. Brooklyn, NY: Polytechnic Press, 1965: 29-39. 